PROCEEDINGS OF THE AMERICAN MATHEMATICAL SOCIETY

Volume 126, Number 3, March 1998, Pages 661-667

S $0002-9939(98) 04127-6$

\title{
EXAMPLES OF CHAIN DOMAINS
}

\author{
R. MAZUREK AND E. ROSZKOWSKA \\ (Communicated by Ken Goodearl)
}

\begin{abstract}
Let $\gamma$ be a nonzero ordinal such that $\alpha+\gamma=\gamma$ for every ordinal $\alpha<\gamma$. A chain domain $R$ (i.e. a domain with linearly ordered lattices of left ideals and right ideals) is constructed such that $R$ is isomorphic with all its nonzero factor-rings and $\gamma$ is the ordinal type of the set of proper ideals of $R$. The construction provides answers to some open questions.
\end{abstract}

\section{INTRODUCTION}

In [6] Leavitt and van Leeuwen studied the class $\mathcal{K}$ of rings which are isomorphic with all nonzero factor-rings. They proved that for every ring $R \in \mathcal{K}$ the set of ideals of $R$ is well-ordered and represented by a prime component ordinal $\gamma$ (i.e. $\gamma>0$ and $\alpha+\gamma=\gamma$ for every ordinal $\alpha<\gamma$ ).

In this paper we show that the class $\mathcal{K}$ contains remarkable examples of rings. Namely, for every prime component ordinal $\gamma$ we construct a chain domain $R$ (i.e. a domain whose lattice of left ideals as well as the lattice of right ideals are linearly ordered) such that $R \in \mathcal{K}$ and $\gamma$ is the ordinal type of the set of proper ideals of $R$. We begin the construction by selecting a semigroup that has properties analogous to those we want $R$ to possess ( $(2)$. The desired ring $R$ is the Jacobson radical of a localization of a semigroup ring of the selected semigroup with respect to an Ore set (§3). In $\S 4$ we use the construction to get answers to five open questions.

All rings in the paper are associative, but do not necessarily have unity. A domain is a ring without zero-divisors. To denote that $I$ is an ideal of a ring or a semigroup $R$ we write $I \triangleleft R$. An ideal $I$ of $R$ is called proper if $I \neq R$. The Jacobson radical of a ring $R$ is denoted by $J(R)$. The sign $\subseteq$ stands for inclusion and $\subset$ for strict inclusion. The minimal element of an ordered nonempty set $S$ is denoted by $\min S$. If $n$ is a positive integer, then $\mathbf{Z}_{n}$ is the ring of integers modulo $n$. When we write $\mathbf{Z}_{p}$, we mean that $p$ is a prime.

\section{Semigroups}

A group $G$ is left ordered if it is nontrivial and supplied with a linear ordering $\leq$ such that $a \leq b$ implies $c a \leq c b$ for all $a, b, c \in G$. If $G$ is a left ordered group and $e$ is the identity of $G$, then the set $G_{+}=\{g \in G \mid e \leq g\}$ of nonnegative elements of $G$ is a subsemigroup of $G$ and the lattice of left ideals as well as the lattice of right ideals of $G_{+}$are linearly ordered. An ideal $H$ of the semigroup $G_{+}$is called

Received by the editors December 1, 1995 and, in revised form, August 27, 1996.

1991 Mathematics Subject Classification. Primary 16D15, 16D25; Secondary 16 N80.

(C)1998 American Mathematical Society 
completely prime if $G_{+} \backslash H$ is a subsemigroup of $G_{+}$. The largest proper ideal of $G_{+}$is equal to $G_{+} \backslash\{e\}$ and denoted by $J\left(G_{+}\right)$.

Let $G$ be a left ordered group with the identity $e$ and let $\gamma$ be a prime component ordinal. The direct product of $\gamma$ copies of the group $G$ is denoted by $G^{\gamma}$ (i.e. $G^{\gamma}=\prod_{0 \leq \alpha<\gamma} G$ ). If $a \in G^{\gamma}$, then for every $\alpha<\gamma$ the $\alpha$ th coordinate of $a$ is denoted by $\pi_{\alpha}(a)$ and $\operatorname{supp}(a)=\left\{\alpha<\gamma \mid \pi_{\alpha}(a) \neq e\right\}$ is the support of $a$. The group $G^{\gamma}$ is left ordered by the lexicographical ordering (i.e. $a \in G_{+}^{\gamma}$ if and only if $a$ is the identity of $G^{\gamma}$ or $e<\pi_{\alpha}(a)$, where $\left.\alpha=\min \operatorname{supp}(a)\right)$. If $\gamma>1$, then for every $0<\alpha<\gamma$ we define $H_{\alpha}=\left\{a \in G_{+}^{\gamma} \mid \exists_{\beta<\alpha} \pi_{\beta}(a) \neq e\right\}$ (for $\gamma=1$ there is no such $\alpha)$.

Lemma 1. If $\gamma>1$, then

a) $H_{\alpha}$ is a completely prime ideal of $G_{+}^{\gamma}, H_{\alpha} \subset J\left(G_{+}^{\gamma}\right)$ and $G_{+}^{\gamma} \simeq G_{+}^{\gamma} \backslash H_{\alpha}$;

b) Every proper ideal $H$ of $J\left(G_{+}^{\gamma}\right)$ is contained in $H_{\alpha}$ for some $\alpha$.

Proof. a) Clearly, the set $H_{\alpha}$ is nonempty and strictly contained in $J\left(G_{+}^{\gamma}\right)$. Let $a \in H_{\alpha}, b \in G_{+}^{\gamma}$ and $\mu=\min (\operatorname{supp}(a) \cup \operatorname{supp}(b))$. Then $\pi_{\mu}(a) \geq e, \pi_{\mu}(b) \geq e$ and at least one of the inequalities is strict. Hence $\pi_{\mu}(a b)=\pi_{\mu}(a) \pi_{\mu}(b)>e$, $\pi_{\mu}(b a)>e$ and $\mu<\alpha$, which shows that $H_{\alpha}$ is an ideal of $G_{+}^{\gamma}$. Since $C=G_{+}^{\gamma} \backslash H_{\alpha}=$ $\left\{a \in G_{+}^{\gamma} \mid \forall_{\beta<\alpha} \pi_{\beta}(a)=e\right\}$ is a subsemigroup of $G_{+}^{\gamma}$, the ideal $H_{\alpha}$ is completely prime. Since $\gamma$ is a prime component ordinal, the map $C \ni a \stackrel{\lambda}{\mapsto} a^{\lambda} \in G_{+}^{\gamma}$, where $\pi_{\beta}\left(a^{\lambda}\right)=\pi_{\alpha+\beta}(a)$ for all $\beta<\gamma$, is a semigroup isomorphism.

b) Since the set of ideals of $J\left(G_{+}^{\gamma}\right)$ is linearly ordered by inclusion, the result follows from the equality $J\left(G_{+}^{\gamma}\right)=\bigcup_{\alpha<\gamma} H_{\alpha}$.

Example 2. Let $\mathbf{R}$ be the set of real numbers and $G$ the set of all functions $f: \mathbf{R} \rightarrow \mathbf{R}$ of the form $f(x)=a x+b$, where $a, b$ are rational numbers and $a>0$. Then $G$ is a group under composition of functions. Let $\varepsilon$ be a positive irrational number. Then the group $G$ is left ordered with respect to the ordering: $f_{1} \leq f_{2}$ if and only if $f_{1}(\varepsilon) \leq f_{2}(\varepsilon)$. In [4, p. 54] it was shown that the only ideal of $J\left(G_{+}\right)$ is $J\left(G_{+}\right)$itself. We will use this to show that ideals of the form $H_{\alpha}$ are the only ideals of the semigroup $G_{+}^{\gamma}$ strictly contained in $J\left(G_{+}^{\gamma}\right)$.

Let $H \subset J\left(G_{+}^{\gamma}\right)$ be an ideal of $G_{+}^{\gamma}$. Then $\gamma>1$ and thus, by Lemma $1 \mathrm{~b}$ ), there exists $\alpha>0$ such that $H \subseteq H_{\alpha}$. Let $\alpha$ be the smallest ordinal for which this inclusion holds. We will show that $H=H_{\alpha}$. Suppose that $H \neq H_{\alpha}$. Then $\alpha$ is not a limit ordinal, say $\alpha=\beta+1$, and $H_{\beta} \subset H \subset H_{\alpha}$ (in case $\alpha=1$ we set $H_{\beta}=H_{0}=\varnothing$ ). Furthermore, $H \backslash H_{\beta} \triangleleft H_{\alpha} \backslash H_{\beta}$ and consequently $\pi_{\beta}\left(H \backslash H_{\beta}\right) \triangleleft \pi_{\beta}\left(H_{\alpha} \backslash H_{\beta}\right)=J\left(G_{+}\right)$. We know that $J\left(G_{+}\right)$has no proper ideal and thus $\pi_{\beta}\left(H \backslash H_{\beta}\right)=J\left(G_{+}\right)$. By assumption we can choose $a \in H_{\alpha}$ with $a \notin H$. Since $\pi_{\beta}(a) \in J\left(G_{+}\right)$and $J\left(G_{+}\right)=$ $\pi_{\beta}\left(H \backslash H_{\beta}\right)$ has no minimal element (otherwise elements greater than the minimal element would form a proper ideal of $J\left(G_{+}\right)$), there exists $h \in H \backslash H_{\beta}$ with $\pi_{\beta}(h)<$ $\pi_{\beta}(a)$. Now we have $h<a$, and so $h^{-1} a \in G_{+}^{\gamma}$. Thus $a=h\left(h^{-1} a\right) \in H G_{+}^{\gamma} \subseteq H$ and this contradiction shows that $H=H_{\alpha}$.

Let us observe that the set $N$ of all functions $f \in G$ of the form $f(x)=x+b$ is a normal subgroup of $G, N$ is isomorphic with the additive group of rational numbers and $G / N$ is isomorphic with the multiplicative group of positive rational numbers. Hence $N$ and $G / N$ are torsion-free abelian. From this it follows that $N^{\gamma}$ is a normal subgroup of $G^{\gamma}$ such that $N^{\gamma}$ and $G^{\gamma} / N^{\gamma}$ are torsion-free abelian. 


\section{Chain domains}

Throughout this section $F$ is a field and $G$ is a left ordered group which has a normal subgroup $N$ such that $N$ and $G / N$ are torsion-free abelian. The semigroup ring of $G_{+}$over $F$ is denoted by $F\left[G_{+}\right]$, i.e. $F\left[G_{+}\right]$consists of all functions $r: G_{+} \rightarrow$ $F$ with finite $\operatorname{support} \operatorname{supp}(r)=\left\{g \in G_{+} \mid r(g) \neq 0\right\}$. Let $S=\left\{s \in F\left[G_{+}\right] \mid e \in\right.$ $\operatorname{supp}(s)\}$.

Lemma 3. If $H \triangleleft G_{+}, r \in F\left[G_{+}\right]$and $s \in S$, then the following conditions are equivalent:

i) $\operatorname{supp}(r) \subseteq H$,

ii) $\operatorname{supp}(r s) \subseteq H$,

iii) $\operatorname{supp}(s r) \subseteq H$.

Proof. The case $r=0$ is obvious, so we assume $r \neq 0$. Clearly i) implies ii). Now assume ii) and let $m=\min \operatorname{supp}(r)$. Since $e \in \operatorname{supp}(s), m=\min \operatorname{supp}(r s)$ and thus $m \in H$. For every $g \in \operatorname{supp}(r)$ we have $m \leq g$, and so $g=m\left(m^{-1} g\right) \in H G_{+} \subseteq H$. Hence $\operatorname{supp}(r) \subseteq H$, i.e. i) holds. The equivalence i) $\Leftrightarrow$ iii) can be proven similarly, by replacing the left ordering $\leq$ by the ordering $\leq^{\prime}$ defined as follows: $a \leq{ }^{\prime} b$ if and only if $b^{-1} \leq a^{-1}$ (in this case $a \leq^{\prime} b$ implies $a c \leq^{\prime} b c$ for all $a, b, c \in G$ ).

By $[1$, Theorems 3.4, 3.6] the set $S$ is a left and right Ore set in the domain $F\left[G_{+}\right]$and the left quotient ring of $F\left[G_{+}\right]$relative to $S$ exists. The quotient ring will be denoted by $F\left(G_{+}\right)$; thus every element of $F\left(G_{+}\right)$can be written as $s^{-1} r$, where $s \in S$ and $r \in F\left[G_{+}\right]$. Let us note that $F\left[G_{+}\right]$and, in particular, $F$ can be regarded as subrings of $F\left(G_{+}\right)$, and $G_{+}$as a subsemigroup of $F\left(G_{+}\right)$.

Proposition 4. a) $F\left(G_{+}\right)$is a chain domain and an $F$-algebra.

b) The map $H \mapsto I^{H}=\left\{s^{-1} r \in F\left(G_{+}\right) \mid \operatorname{supp}(r) \subseteq H\right\}$ defines an inclusionpreserving bijection of the set of all ideals of the semigroup $G_{+}$with the set of all nonzero ideals of the ring $F\left(G_{+}\right)$.

c) If $H$ is a completely prime ideal of $G_{+}$and $G_{+} \simeq G_{+} \backslash H$, then $F\left(G_{+}\right) \simeq$ $F\left(G_{+}\right) / I^{H}$.

d) $J\left(F\left(G_{+}\right)\right)=\left\{s^{-1} r \in F\left(G_{+}\right) \mid e \notin \operatorname{supp}(r)\right\}$ and $F\left(G_{+}\right) / J\left(F\left(G_{+}\right)\right) \simeq F$.

Proof. a) $F\left(G_{+}\right)$is a chain domain by [1, Corollary 3.7]. Since $F$ is contained in the center of $F\left(G_{+}\right)$, the ring $F\left(G_{+}\right)$is an algebra over $F$.

b) Let $H$ be an ideal of $G_{+}$. Assume that $s^{-1} r=s_{1}^{-1} r_{1} \in F\left(G_{+}\right)$and $\operatorname{supp}(r) \subseteq$ $H$. Then $a r=b r_{1}$ for some $a, b \in S$ and consequently, by Lemma $3, \operatorname{supp}\left(r_{1}\right) \subseteq H$. This shows that the set $I^{H}$ is well defined.

Now we show that $I^{H}$ is a nonzero ideal of $F\left(G_{+}\right)$. Since $I=\left\{r \in F\left[G_{+}\right] \mid \operatorname{supp}(r)\right.$ $\subseteq H\}$ is a nonzero ideal of $F\left[G_{+}\right]$, it follows that $I^{H}=\left\{s^{-1} r \in F\left(G_{+}\right) \mid r \in I\right\}=$ $\bar{F}\left(G_{+}\right) I$ is a nonzero left ideal of $F\left(G_{+}\right)$. To show that $I^{H}$ is a right ideal of $F\left(G_{+}\right)$, let $r \in I$ and $s \in S$. Since $S$ is a left Ore set in $F\left[G_{+}\right]$, there exist $r_{1} \in F\left[G_{+}\right]$and $s_{1} \in S$ such that $s_{1} r=r_{1} s$. By Lemma $3, \operatorname{supp}\left(r_{1}\right) \subseteq H$ and thus $r_{1} \in I$. Now, using $r s^{-1}=s_{1}^{-1} r_{1}$, we establish that $I^{H}=F\left(G_{+}\right) I$ is a right ideal of $F\left(G_{+}\right)$.

We show that the map $H \mapsto I^{H}$ is injective. Let $H_{1}, H_{2}$ be ideals of $G_{+}$with $I^{H_{1}}=I^{H_{2}}$ and let $g \in H_{1}$. Then $g \in I^{H_{2}}$, and so $g=s^{-1} r$ for some $s \in S$ and $r \in F\left[G_{+}\right]$with $\operatorname{supp}(r) \subseteq H_{2}$. Hence $g \in \operatorname{supp}(s g)=\operatorname{supp}(r) \subseteq H_{2}$. This shows that $H_{1} \subseteq H_{2}$ and analogous arguments give the opposite inclusion.

We show that the map $H \mapsto I^{H}$ is surjective. Let $I$ be a nonzero ideal of $F\left(G_{+}\right)$ and let $H=I \cap G_{+}$. Since the set $H$ is nonempty (we will see this in a moment) and 
$G_{+}$is a subsemigroup of $F\left(G_{+}\right)$and $I$ is an ideal of the multiplicative semigroup $F\left(G_{+}\right)$, it follows that $H$ is an ideal of $G_{+}$. Now we prove that $I=I^{H}$. Let $0 \neq s^{-1} r \in I$ and let $m=\min \operatorname{supp}(r)$. Then $r=m s_{1}$ for some $s_{1} \in S$ and so $m=s\left(s^{-1} r\right) s_{1}^{-1} \in I$. Hence $m \in H$ (in particular, $H \neq \varnothing$ ) and consequently $\operatorname{supp}(r) \subseteq H$. Thus $s^{-1} r \in I^{H}$, which shows that $I \subseteq I^{H}$. To get the opposite inclusion, let $s^{-1} r \in I^{H}$. Then $\operatorname{supp}(r) \subseteq H=I \cap G_{+}$, and thus $r \in I$. Hence $s^{-1} r \in F\left(G_{+}\right) I \subseteq I$, so $I^{H} \subseteq I$.

c) Let $H$ be a completely prime ideal of $G_{+}$and let $\phi: G_{+} \rightarrow G_{+} \backslash H$ be a semigroup isomorphism. Recall that $F\left[G_{+}\right]$consists of all functions $r: G_{+} \rightarrow F$ with finite support. For every $r \in F\left[G_{+}\right]$we put $\psi(r)=r \circ \phi$, the composition of $\phi$ and $r$. Then $\psi: F\left[G_{+}\right] \rightarrow F\left[G_{+}\right]$is a ring epimorphism and $\psi(S)=S$. Hence, putting $\eta\left(s^{-1} r\right)=\psi(s)^{-1} \psi(r)$, we get a ring epimorphism $\eta: F\left(G_{+}\right) \rightarrow F\left(G_{+}\right)$. Moreover, $\eta\left(s^{-1} r\right)=0$ if and only if $\psi(r)=r \circ \phi=0$. The latter is equivalent to $\operatorname{supp}(r) \subseteq H$. Hence $\operatorname{ker} \eta=I^{H}$ and therefore $F\left(G_{+}\right) \simeq F\left(G_{+}\right) / I^{H}$.

d) By a) $F\left(G_{+}\right)$is a chain domain and thus $J\left(F\left(G_{+}\right)\right)$is the largest proper ideal of $F\left(G_{+}\right)$. Hence, by b), $J\left(F\left(G_{+}\right)\right)$corresponds to $J\left(G_{+}\right)=G_{+} \backslash\{e\}$, which proves the first part of d). Since the map $\varphi: F\left(G_{+}\right) \rightarrow F, \varphi\left(s^{-1} r\right)=s(e)^{-1} r(e)$, is a ring epimorphism and $\operatorname{ker} \varphi=J\left(F\left(G_{+}\right)\right)$, we get the second part of d).

Lemma 5. Let $A$ be a ring with unity and $R$ be an ideal of $A$ such that $A / R \simeq \mathbf{Z}_{n}$ for some positive integer $n$. If $L$ is a left ideal of $R$, then $L$ is a left ideal of $A$.

Proof. Let $a \in A$. Since $A / R \simeq \mathbf{Z}_{n}, a=m \cdot 1+r$ for some positive integer $m$ and $r \in R$. Hence

$$
a L=(m \cdot 1+r) L \subseteq m L+r L \subseteq L .
$$

Thus $L$ is a left ideal of $A$.

Let $\gamma$ be a prime component ordinal and $F=\mathbf{Z}_{p}$. Our assumptions about $G$ (see the beginning of this section) imply that the group $G^{\gamma}=\prod_{0 \leq \alpha<\gamma} G$ is left ordered by the lexicographical ordering and $G^{\gamma}$ has a normal subgroup $N^{\gamma}$ with $G^{\gamma} / N^{\gamma}$ and $N^{\gamma}$ torsion-free abelian. Hence, by Proposition 4 a), $F\left(G_{+}^{\gamma}\right)$ is a chain domain. Let $R=J\left(F\left(G_{+}^{\gamma}\right)\right)$. Since by Proposition 4 d) $F\left(G_{+}^{\gamma}\right) / R \simeq \mathbf{Z}_{p}$, Lemma 5 implies that $R$ is a chain domain. Moreover, by Lemma 5 , the set of ideals of $R$ coincides with the set of those ideals of $F\left(G_{+}^{\gamma}\right)$ which are contained in $J\left(F\left(G_{+}^{\gamma}\right)\right)$. Hence, if $I$ is a nonzero proper ideal of $R$, then by Proposition $4 \mathrm{~b}$ ) and $\mathrm{d}$ ), there exists a proper ideal $H$ of the semigroup $J\left(G_{+}^{\gamma}\right)$ such that $I=I^{H}$. Assume in addition that $\gamma>1$. Then, by Lemma $1 \mathrm{~b}), H \subseteq H_{\alpha}$ for some $\alpha<\gamma$. We know from Lemma 1 a) that $G_{+}^{\gamma} \simeq G_{+}^{\gamma} \backslash H_{\alpha}$ and thus by Proposition $\left.4 \mathrm{c}\right), F\left(G_{+}^{\gamma}\right) \simeq F\left(G_{+}^{\gamma}\right) / I^{H_{\alpha}}$. Since $R$ is the Jacobson radical of $F\left(G_{+}^{\gamma}\right)$, it follows that $R \simeq R / I^{H_{\alpha}}$ and consequently $R / I$ can be homomorphically mapped onto $R$, because $I=I^{H} \subseteq I^{H_{\alpha}}$. We have proved

Corollary 6. Let $\gamma$ be a prime component ordinal, $F=\mathbf{Z}_{p}$ and $R=J\left(F\left(G_{+}^{\gamma}\right)\right)$. Then

a) $R$ is a chain domain and every nonzero proper ideal of $R$ has the form $I^{H}$ for some proper ideal $H$ of $J\left(G_{+}^{\gamma}\right)$;

b) If $\gamma>1$, then $R / I^{H_{\alpha}} \simeq R$ for every nonzero ordinal $\alpha<\gamma$, and every nonzero homomorphic image of $R$ can be homomorphically mapped onto $R$. 


\section{Applichtions}

We will use the above construction to get answers to some open questions.

In [6] Leavitt and van Leeuwen studied the class $\mathcal{K}$ of all rings $R$ such that $R / I$ is isomorphic with $R$ for every proper ideal $I$ of $R$. They proved that if $R \in \mathcal{K}$, then for some prime component ordinal $\gamma$ the set of all ideals of $R$ can be written as $\left\{I_{\alpha}\right\}_{0 \leq \alpha \leq \gamma}$, where $I_{\alpha} \subset I_{\beta}$ if and only if $0 \leq \alpha<\beta \leq \gamma$.

Leavitt and van Leeuwen ([6, Remark 3]) asked the following question: Does there exist a ring $R \in \mathcal{K}$ with $R=I_{\gamma}$ for some $\gamma>\omega$ ? We will show that for every prime component ordinal $\gamma$ there exists a chain domain $R \in \mathcal{K}$ with $R=I_{\gamma}$. In particular, we will get a positive answer to the above question.

Example 7. Let $\gamma$ be a prime component ordinal, $G$ be the group described in Example 2 and $F=\mathbf{Z}_{p}$. Then the ideals $H_{\alpha}$, where $0<\alpha<\gamma$, are the only proper ideals of $J\left(G_{+}^{\gamma}\right)$. Now Corollary 6 shows that $R=J\left(F\left(G_{+}^{\gamma}\right)\right)$ is a chain domain, $R \in \mathcal{K}$ and $\gamma$ is the order type of the set of proper ideals of $R$.

In [11] Watters asked the following question (Question 1, p. 181): Does there exist a Jacobson radical $F$-algebra $R$ over a field $F$ such that $R$ does not satisfy the maximum condition on $F$-ideals, but each proper $F$-ideal of $R$ satisfies the maximum condition on $F$-ideals? Here $F$-ideal means both $F$-module and ideal in the ring-theoretic sense. We will show that the answer to the question is positive.

Let $R$ be the ring constructed in Example 7 for $\gamma=\omega$. Then $R$ is an $F$-algebra, all ideals of $R$ are $F$-ideals and they form a chain

$$
0=I_{0} \subset I_{1} \subset \cdots \subset I_{n} \subset \cdots \subset I_{\omega}=R .
$$

Hence $R$ is a Jacobson radical $F$-algebra that does not satisfy the maximum condition on $F$-ideals. Now, if $I$ is a proper ideal of $R$, then $I=I_{n}$ for some nonnegative integer $n$. Since for every $\alpha<\gamma, R / I_{\alpha} \simeq R$ is a domain, it follows that all ideals of $R$ are prime. Thus Andrunakievich's lemma (if $K \triangleleft I \triangleleft R$ and $T$ is the ideal of $R$ generated by $K$, then $T^{3} \subseteq K$ ) implies that the only ideals of $I$ are $I_{0}, I_{1}, \ldots, I_{n}$. Hence every proper ideal of $R$ satisfies the maximum condition on ideals.

Now we pass to questions connected with atoms of lattices of radicals. The lattice of all radicals will be denoted by $\mathbf{L}$ and the lattice of left strong radicals by Ls. Recall ([9]) that a nonzero radical $\mathcal{P}$ is an atom of $\mathbf{L}(\mathbf{L s})$ if and only if for every $0 \neq R \in \mathcal{P}, \mathcal{P}=l_{R}\left(\mathcal{P}=l s_{R}\right)$, where $l_{R}\left(l_{s_{R}}\right)$ denotes the lower (lower left strong) radical determined by $R$. If $R$ is a nonzero idempotent ring, then $A \in l_{R}\left(A \in l s_{R}\right)$ if and only if every nonzero homomorphic image of $A$ contains a nonzero ideal (left ideal) which is a homomorphic image of $R$. Basic facts about radicals can be found in [2] and about their lattices in [10].

We will show that the ring constructed in Example 7 generates atoms of $\mathbf{L}$ and Ls. We will need the following

Lemma 8. Suppose $R$ is a chain domain and the characteristic of $R$ is a prime $p$. If $R$ is a left ideal of a Jacobson radical ring A, then $R$ is a homomorphic image of $A$.

Proof. Let $0 \neq r \in R$. Since $A=J(A), r \notin A r$ and thus $R r+\mathbf{Z}_{p} r \nsubseteq A r$. Since all left ideals of $R$ form a chain, we get $A r \subseteq R r+\mathbf{Z}_{p} r$. Let $a \in A$ be arbitrary. Then $a r=s r+n r$ for some $s \in R$ and $n \in \mathbf{Z}_{p}$. But $n r=0$ (if not, then $n \neq 0$, so $n$ is invertible in $\mathbf{Z}_{p}$ and we get $r \in A r$, a contradiction), and thus $a r=s r$. Since $R$ is a domain, $s$ is uniquely determined; denote this element by $f(a)$. Clearly, 
the map $A \ni a \stackrel{f}{\mapsto} f(a) \in R$ is an epimorphism of additive groups. We claim that $f\left(a_{1} a_{2}\right)=f\left(a_{1}\right) f\left(a_{2}\right)$. This is obvious if $a_{2} r=0$, so we assume $a_{2} r \neq 0$. Since right ideals of $R$ are linearly ordered by inclusion, we have $a_{2} r R \subseteq r R$ or $r R \subseteq a_{2} r R$. In both cases there exist $t, w \in R \backslash\{0\}$ such that $a_{2} r t=r w$. Since $\left(f\left(a_{1} a_{2}\right)-f\left(a_{1}\right) f\left(a_{2}\right)\right) r t=0$ and $r t \neq 0, f\left(a_{1} a_{2}\right)-f\left(a_{1}\right) f\left(a_{2}\right)=0$ and this proves our claim. Thus $R$ is a homomorphic image of $A$.

Let $R$ be the ring constructed in Example 7 . We claim that $l s_{R}$ is an atom of Ls. Let $0 \neq A \in l s_{R}$. Then $A$ contains a nonzero left ideal isomorphic to $R$. Since $A \in l s_{R}$ and $R=J(R)$, it follows that $A=J(A)$ and thus, by Lemma $8, R$ is a homomorphic image of $A$. Hence $R \in l s_{A}$, which proves the claim. Analogous arguments show that $l_{R}$ is an atom of $\mathbf{L}$.

In [8] Puczyłowski and Roszkowska asked the following question (Question 3): Does there exist a simple ring without unity such that $l s_{R}$ is an atom of the lattice Ls? Since for $\gamma=1$ the ring $R$ from Example 7 is a simple ring without unity and, by the preceding paragraph, $l s_{R}$ is an atom of $\mathbf{L s}$, we get a positive answer to this question.

In [5] Gardner introduced the following property of an idempotent simple ring $P$ :

$(\rho) \quad$ if $P$ is an ideal of a $\operatorname{ring} Q$ and $Q / P \simeq P$, then $Q \simeq P \oplus P$.

Gardner proved that if an idempotent simple ring $P$ satisfies $(\rho)$, then $l_{P}$ is an atom of $\mathbf{L}$.

Puczyłowski and Roszkowska asked the question ([8, Question 1]): Does there exist a simple domain (without unity) not satisfying $(\rho)$ ? Here we answer this question in the affirmative. Namely, let $R$ be the ring constructed in Example 7 for $\gamma>1$. Then all ideals of $R$ form a chain $0=I_{0} \subset I_{1} \subset I_{2} \subset \cdots \subset I_{\gamma}=R$. Since $R$ is isomorphic with all its nonzero factor-rings, $l_{R} \subseteq l_{I_{1}}$. Since $R$ is a domain, the Andrunakievich lemma shows that $I_{1}$ is a simple domain. Clearly, $I_{1} \in l_{I_{1}} \backslash I_{R}$. Thus $l_{R} \subset l_{I_{1}}$, so $l_{I_{1}}$ is not an atom of $\mathbf{L}$ and consequently, by Gardner's result, $I_{1}$ does not satisfy $(\rho)$.

Let $R$ be a commutative and idempotent chain ring (a commutative ring is a chain ring provided its ideals are linearly ordered by inclusion). In [9] it was proved that $l_{R}$ is an atom of $\mathbf{L}$ if and only if every nonzero homomorphic image of $R$ can be homomorphically mapped onto $R$. All known examples of rings of this type not being fields were nil rings. Thus Puczyłowski asked the question ([7, Question 6]): Does there exist a commutative and idempotent chain ring $R$ which is neither nil nor a field and such that every nonzero homomorphic image of $R$ can be homomorphically mapped onto R?

We show the answer to the question is positive. Let $G$ be an abelian ordered group, $\gamma>1$ be a prime component ordinal and $F=\mathbf{Z}_{p}$. Then clearly $R=$ $J\left(F\left(G_{+}^{\gamma}\right)\right)$ is not a field. Moreover, by Corollary $6, R$ is a commutative chain domain such that every nonzero homomorphic image of $R$ can be homomorphically mapped onto $R$.

Remarks. 1) For $\gamma=1$ and $p=2$ the ring $R$ from Example 7 is the simple Jacobson radical chain domain constructed by Dubrovin in [4].

2) All previously known examples of atoms of $\mathbf{L}$ or $\mathbf{L} \mathbf{s}$ have contained a simple ring or a commutative ring. Let us note that if $R$ is the ring constructed in Example 7 for $\gamma>1$, then the atoms $l_{R}$ and $l s_{R}$ do not have this property. 
3) To get a commutative chain ring which has properties described in the question of Puczyłowski, one can also start with an abelian ordered group $G$, apply the Malcev-Neumann construction ([3]) to the group $G^{\gamma}(\gamma>1)$ and the field $\mathbf{Z}_{p}$, and take the Jacobson radical of the resulting ring.

\section{ACKNOWLEDGEMENTS}

Some results in this paper were part of the first author's dissertation, and some other results were part of the second author's dissertation, both done under the supervision of Professor E. R. Puczyłowski at Warsaw University. The authors would like to thank Professor Puczyłowski for his help and encouragement.

\section{REFERENCES}

1. U. Albrecht and G. Törner, Group rings and generalized valuations, Comm. Algebra 12 (1984), 2243-2272. MR 85f: 16013

2. V. A. Andrunakievich and J. M. Ryabukhin, Radicals of algebras and structural theory (Russian), Nauka, Moscow, 1979. MR 82a:16001

3. P. M. Cohn, Free rings and their relations, London Math. Soc. Monographs No. 19, Academic Press, London, 1985. MR 87e:16006

4. N. I. Dubrovin, Chain domains (Russian), Vestnik Moscov. Univ. Ser. I Mat. Meh. 1980, no. 2, 51-54. MR 81g:16004

5. B. J. Gardner, Simple rings whose lower radicals are atoms, Acta Math. Hungar. 43 (1984), 131-135. MR 85a:16007

6. W. G. Leavitt and L. C. A. van Leeuwen, Rings isomorphic with all proper factor-rings, Ring theory (Proc. 1978 Antwerp Conf.), Marcel Dekker, New York and Basel, 1979, 783-798. MR 81i: 16010

7. E. R. Puczyłowski, Some questions concerning radicals of associative rings, Theory of Radicals (Proc. Conf. Szekszard, 1991), 209-227, Colloq. Math. Soc. János Bolyai, Vol. 61, NorthHolland, Amsterdam, 1993. MR 94j:16033

8. E. R. Puczyłowski and E. Roszkowska, Atoms of lattices of radicals of associative rings, Radical Theory (Proc. Conf. Sendai, 1988), 123-134. MR 90e:16009

9. $\quad$ On atoms and coatoms in lattices of radicals of associative rings, Comm. Algebra 20 (1992), 955-977. MR 93e:16032

10. R. L. Snider, Lattices of radicals, Pacific J. Math. 40 (1972), 207-220. MR 46:7290

11. J. F. Watters, Noncommutative minimally non-Noetherian rings, Math. Scand. 40 (1977), 176-182. MR 56:15694

Institute of Mathematics, University of Warsaw, Biąystok Division, Akademicka 2, 15-267 Biąystok, Poland

E-mail address: mazurek@cksr.ac.bialystok.pl

Faculty of Economy, University of Warsaw, Bialystok Division, Sosnowa 62, 15-887 BiaŁystok, Poland

Current address: Faculty of Economy, University in Białystok, Warszawska 63, 15-062

Białystok, Poland 